\title{
Respiratory Motion Correction in Oncologic PET Using T1-Weighted MR Imaging on a Simultaneous Whole-Body PET/MR System
}

\author{
Christian Würslin ${ }^{1}$, Holger Schmidt ${ }^{1,2}$, Petros Martirosian ${ }^{3}$, Cornelia Brendle ${ }^{1}$, Andreas Boss ${ }^{1,4}$, Nina F. Schwenzer ${ }^{1}$, \\ and Lars Stegger ${ }^{1,5}$ \\ ${ }^{I}$ Diagnostic and Interventional Radiology, Department of Radiology, University of Tübingen, Tübingen, Germany; ${ }^{2}$ Laboratory \\ for Preclinical Imaging and Imaging Technology of the Werner Siemens-Foundation, Department of Preclinical Imaging and \\ Radiopharmacy, Department of Radiology, University of Tübingen, Tübingen, Germany; ${ }^{3}$ Section on Experimental Radiology, \\ Department of Radiology, University of Tübingen, Tübingen, Germany; ${ }^{4}$ Department of Radiology, University Hospital Zürich, \\ Zürich, Switzerland; and ${ }^{5}$ Department of Nuclear Medicine and European Institute for Molecular Imaging, University of \\ Münster, Münster, Germany
}

Hybrid PET/MR combines the exceptional molecular sensitivity of PET with the high resolution and versatility of MR imaging. Simultaneous data acquisition additionally promises the use of MR to enhance the quality of PET images, for example, by respiratory motion correction. This advantage is especially relevant in thoracic and abdominal areas to improve the visibility of small lesions with low radiotracer uptake and to enhance uptake quantification. In this work, the applicability and performance of an MR-based method of respiratory motion correction for PET tumor imaging was evaluated in phantom and patient studies. Methods: PET list-mode data from a motion phantom with ${ }^{22} \mathrm{Na}$ point sources and 5 patients with tumor manifestations in the thorax and upper abdomen were acquired on a simultaneous hybrid PET/MR system. During the first 3 min of a 5-min PET scan, the respiration-induced tissue deformation in the PET field of view was recorded using a sagittal 2-dimensional multislice gradient echo MR sequence. MR navigator data to measure the location of the diaphragm were acquired throughout the PET scan. Respiration-gated PET data were coregistered using the MR-derived motion fields to obtain a single motion-corrected PET dataset. The effect of motion correction on tumor visibility, delineation, and radiotracer uptake quantification was analyzed with respect to uncorrected and gated images. Results: Image quality in terms of lesion delineation and uptake quantification was significantly improved compared with uncorrected images for both phantom and patient data. In patients, in head-feet line profiles of 14 manifestations, the slope became steeper by $66.7 \%(P=0.001)$ and full width at half maximum was reduced by $20.6 \%(P=0.001)$. The mean increase in maximum standardized uptake value, lesion-to-background ratio (contrast), and signal-to-noise ratio was $28.1 \%(P=0.001), 24.7 \%(P=0.001)$, and $27.3 \%(P=0.003)$, respectively. Lesion volume was reduced by an average of $26.5 \%(P=0.002)$. As opposed to the gated images, no increase in background noise was observed.

Received Mar. 14, 2012; revision accepted Sep. 17, 2012.

For correspondence or reprints contact: Holger Schmidt, Diagnostic and Interventional Radiology, University of Tübingen, Hoppe-Seyler-Strasse 3, 72076 Tübingen, Germany.

E-mail: h.schmidt@med.uni-tuebingen.de

Published online Jan. 3, 2013.

COPYRIGHT (C) 2013 by the Society of Nuclear Medicine and Molecular Imaging, Inc.
However, motion correction performed worse than gating in terms of contrast $(-11.3 \%, P=0.002)$, maximum standardized uptake value $(-10.7 \%, P=0.003)$, and slope steepness $(-19.3 \%, P=0.001)$. Conclusion: The proposed method for MR-based respiratory motion correction of PET data proved feasible and effective. The short examination time and convenience (no additional equipment required) of the method allow for easy integration into clinical routine imaging. Performance compared with gating procedures can be further improved using list-mode-based motion correction.

Key Words: PET; MRI; simultaneous multi-modality imaging; respiratory motion correction

J Nucl Med 2013; 54:464-471

DOI: 10.2967/jnumed.112.105296

$\mathbf{H}$ ybrid PET/MR imaging systems combine the high molecular sensitivity of PET and the superior resolution and versatility of MR imaging for improved tumor imaging (1). Furthermore, systems capable of simultaneous PET/MR data acquisition can improve PET image quality and radiotracer uptake quantification by the use of MR-based correction methods, for example, for respiratory motion.

Respiratory motion adversely affects PET tumor imaging especially in the thorax and upper abdomen. Breath-hold acquisitions are impractical given the long acquisition time on the order of several minutes. The detection of small lesions in tumor staging, the assessment of treatment response by measuring radiotracer uptake, and the definition of tumor volume for external radiation therapy are 3 important areas of tumor imaging hampered by motion (2-5). Furthermore, a misalignment between PET images and images from CT or MR imaging, often acquired during breath-holding, can occur and may interfere with lesion identification and render CT- or MR-derived attenuation maps ( $\mu$ Maps) for attenuation correction invalid (6). Respiratory motion is especially difficult to correct for since it manifests as nonrigid deformations in 
the body with a usual peak amplitude of 15-26 mm and maximal organ movements of up to $40 \mathrm{~mm}$ (head-feet direction) (7-10).

Several approaches have been proposed to compensate for this motion. PET gating, in which counts from only a specific phase of the respiratory cycle are used for image generation, usually around the end-expiratory position (11), is hampered by decreased signal-to-noise ratio (SNR) unless either examination time or the amount of injected activity is increased. More sophisticated approaches aim at maintaining all counts in the data by transforming them to a reference gate (usually end-expiration). These approaches require a description of the respiration-induced deformations in the body. Motion fields can be applied after reconstruction of the individual gates to obtain a single summed and motion-corrected image volume (12-14), or they may already be applied during image reconstruction $(15,16)$. Motion fields may be derived from the PET data themselves if enough anatomic landmarks are visible in the images (12-14); however, this approach is likely to fail for specific radiotracers with little background uptake. Motion fields can also be derived from 4-dimensional (4D) CT data, but at the cost of increased exposure to ionizing radiation (15). Recently, the use of simultaneous PET/MR has been proposed for the generation of motion fields (17), initially to correct for rigid head movement in a brain PET/MR system (18). In the case of nonrigid respiration motion, the use of 2-dimensional (2D) and 3-dimensional (3D) MR sequences for nonrigid motion compensation has been discussed and evaluated using simulated PET data $(19,20)$, phantom studies $(21,22)$, and recently in rabbits and primates $(23)$.

In this work, the applicability and performance of a method for respiratory motion correction in PET tumor imaging with a whole-body PET/MR system was evaluated using phantom and in vivo data. The motion fields were generated from 2D multislice MR data in combination with a 1-dimensional navigator echo sequence. The method was designed to be convenient and time-efficient for use in routine imaging.

\section{MATERIALS AND METHODS}

\section{Study Design}

The proposed MR-based motion correction was evaluated using phantom data and image data from tumor patients. Images corrected for motion were compared with uncorrected images (free-breathing), images obtained from the end-expiratory gate (gated), and images acquired without respiratory motion (phantom study only).

Phantom. A cylindric MR phantom with an acrylic glass cube in its isocenter was equipped with three ${ }^{22} \mathrm{Na}$ point sources (37 kBq/source) and filled with ${ }^{18} \mathrm{~F}$-doped water $(4 \mathrm{kBq} / \mathrm{mL})$ for background activity. One point source was attached to the central cube, and 2 were positioned above and below it (Supplemental Fig. 1; supplemental materials are available online only at http://jnm. snmjournals.org). The phantom was placed on a trolley connected to a motor for harmonic movement (amplitude, $4 \mathrm{~cm}$; frequency, $0.25 \mathrm{~Hz}$ ) along the scanner's $z$-axis to simulate a worst-case scenario for respiratory motion along the head-feet direction.

Patients. Five consecutively enrolled patients with lesions in the thorax or upper abdomen (bronchial carcinoma, $n=3$; colorectal carcinoma, $n=1$; nasopharynx carcinoma $n=1$ ) participated in the study (age range, 52-70 y; mean, $59.8 \pm 8.1$ y). The study was approved by the local ethics committee, and all patients gave written consent. Four patients had lesions affected by motion (patients A-D, Table 1) and other nonmoving osseous lesions; one had only nonmoving lesions. Eight of these nonmoving lesions were evaluated to detect new errors introduced by motion correction (Table 2). PET/MR scanning commenced without repeated radiotracer injection after routine PET/CT $(\sim 240$ min after injection of $336-371 \mathrm{MBq}$ of $\left.{ }^{18} \mathrm{~F}-\mathrm{FDG}\right)$.

TABLE 1

Overview of Evaluated Lesions in 4 Patients (A-D) and Absolute Values of Lesion Parameters Obtained from Gated Images (Reference Standard)

\begin{tabular}{|c|c|c|c|c|c|c|c|c|c|}
\hline Lesion & Location & $\operatorname{Size}^{\star}\left(\mathrm{mm}^{2}\right)$ & $\Delta \mathrm{x}(\mathrm{mm})$ & Contrast & SUV $_{\max }$ & SNR & FWHM (mm) & Slope (1/cm) & Volume $\left(\mathrm{cm}^{3}\right)$ \\
\hline A1 & Lung, middle lobe & $8 \times 7$ & 5.6 & 6.8 & 2.7 & 28.5 & 6.5 & 13.9 & 0.58 \\
\hline $\mathrm{A} 2$ & Lung, lower lobe & $16 \times 6$ & 6.5 & 13.8 & 5.3 & 55.5 & 9.7 & 28.1 & 1.80 \\
\hline A3 & Lung, lower lobe & $12 \times 8$ & 2.7 & 8.4 & 3.5 & 29.2 & 6.7 & 21.8 & 0.85 \\
\hline A4 & Lung, lower lobe & $8 \times 8$ & 10.4 & 14.6 & 6.1 & 35.6 & 7.5 & 31.2 & 0.53 \\
\hline A5 & Lung, lower lobe & $8 \times 5$ & 11.0 & 3.6 & 1.5 & 8.4 & 5.9 & 9.4 & 0.66 \\
\hline A6 & Lymph node, hilar & $11 \times 8$ & 7.2 & 18.7 & 6.8 & 63.8 & 9.7 & 36.5 & 1.27 \\
\hline A7 & Spleen & $21 \times 16$ & 13.7 & 7.2 & 14.3 & 18.2 & 13.2 & 69.9 & 1.65 \\
\hline A8 & Lung, lower lobe & $5 \times 3$ & 7.8 & 4.9 & 2.2 & 17.3 & 5.3 & 10.5 & 0.41 \\
\hline A9 & Lung, lower lobe & $6 \times 4$ & 5.5 & 5.2 & 2.1 & 16.4 & 7.1 & 11.4 & 1.13 \\
\hline A10 & Spleen & $9 \times 8$ & 9.3 & 5.1 & 7.9 & 15.6 & 9.7 & 46.7 & 1.16 \\
\hline B1 & Lung, lower lobe & $13 \times 8$ & 7.4 & 9.1 & 2.8 & 10.4 & 5.5 & 16.2 & 0.31 \\
\hline C1 & Lung, lower lobe & $13 \times 7$ & 13.5 & 8.5 & 4.4 & 26.8 & 6.3 & 23.7 & 0.34 \\
\hline $\mathrm{C} 2$ & Lung, lower lobe & $13 \times 12$ & 16.2 & 6.5 & 4.3 & 24.8 & 8.5 & 25.3 & 0.97 \\
\hline D1 & Lymph node, hilar & $11 \times 10$ & 5.1 & 14.2 & 8.1 & 40.0 & 7.1 & 43.0 & 0.44 \\
\hline
\end{tabular}


TABLE 2

Mean Change of Lesion Parameters in 8 Steady Lesions

\begin{tabular}{clcccccc}
\hline Image & Index & Contrast & SUV $_{\text {Max }}$ & SNR & FWHM & Slope & Volume \\
\hline Uncorrected/gated & Mean & $-0.9 \%$ & $-3.0 \%$ & $31.3 \%$ & $6.4 \%$ & $-12.6 \%$ & $23.1 \%$ \\
& SD & $9.4 \%$ & $5.7 \%$ & $11.6 \%$ & $9.9 \%$ & $6.9 \%$ & $20.1 \%$ \\
& $P$ & 0.726 & 0.159 & $0.012^{*}$ & 0.131 & $0.012^{*}$ & 0.025 \\
Corrected/gated & Mean & $-4.6 \%$ & $-3.9 \%$ & $32.9 \%$ & $5.3 \%$ & $-11.3 \%$ & $31.5 \%$ \\
& SD & $7.8 \%$ & $8.2 \%$ & $13.4 \%$ & $9.6 \%$ & $8.2 \%$ & $29.3 \%$ \\
\hline & $P$ & 0.161 & 0.362 & $0.012^{*}$ & 0.233 & $0.012^{*}$ & 0.042 \\
Corrected/uncorrected & Mean & $-3.6 \%$ & $-1.0 \%$ & $1.4 \%$ & $-0.9 \%$ & $1.6 \%$ & $6.2 \%$ \\
& SD & $5.4 \%$ & $3.5 \%$ & $7.4 \%$ & $3.6 \%$ & $8.0 \%$ & $12.3 \%$ \\
& $P$ & 0.263 & 0.351 & 0.779 & 0.395 & 0.44 & 0.553
\end{tabular}

${ }^{*}$ Statistically significant.

\section{Data Acquisition}

Figure 1 shows the different steps of the proposed motion correction method and the data flow. The dashed signal path corresponds to a standard PET reconstruction; the solid lines depict the data flow of the motion correction. The MR system provides 3 types of information: images serving as the basis for calculation of the patient $\mu$ Map (attenuation for $511-\mathrm{keV}$ photons) used for PET attenuation correction (MR-Dixon scan; not suitable for the phantom) (24), 2D gradient echo images for motion field generation, and navigator images for the extraction of a respiration signal, the latter 2 acquired simultaneously with the PET data.

All data were acquired on a hybrid whole-body PET/MR system (Biograph mMR; Siemens Healthcare). The system combines a PET detector system based on lutetium-oxyorthosilicate scintillators coupled to avalanche photodiodes with a transaxial and axial field of view (FOV) of $59.4 \mathrm{~cm}$ and $25.8 \mathrm{~cm}$, respectively, and a 3-T MR system. Phased-array body coils optimized for minimally 511-keV photon attenuation were used for signal detection.

Phantom Scans. Because the usual MR-Dixon scan for generation of the $\mu$ Map (24) can depict fat and water but not acrylic glass and is therefore unsuitable for the phantom study, a separate CT scan had been previously acquired for the phantom's attenuation map. This was coregistered to the PET data. Simultaneous PET/MR data acquisition (MR: 2D multislice sequence and navigator images; PET: list mode) was performed with the moving phantom using the same imaging parameters as in the patient measurements. Additionally, a PET-only data acquisition without motion (steady phantom) was performed to provide ground-truth data (evidently not possible in patients).
Patient Scans. An MR-Dixon scan was acquired in breath-hold (19 s, end-expiratory position), and tissues were classified to obtain a $\mu$ Map of the PET FOV (24). Then, PET emission data of 1 bed position (FOV covering thorax and upper abdomen) were recorded for 5 min under free breathing in list mode. During the first $3 \mathrm{~min}$ of the PET scan, multiple 2D MR slices covering the patient's body within the PET FOV, as well as the respiratory cycle (giving effectively 4D temporospatial data) for motion estimation, were acquired. A spoiled gradient echo imaging sequence (repetition time, $3.7 \mathrm{~ms}$; echo time, $1.8 \mathrm{~ms}$; flip angle, $15^{\circ}$; bandwidth, $670 \mathrm{~Hz} /$ pixel; matrix size, $192 \times 144$; FOV, $384 \times 288 \mathrm{~mm}^{2}$; 6/8-phase partial Fourier; in-plane resolution, $2 \times 2 \mathrm{~mm}^{2}$; acquisition time per image, $0.4 \mathrm{~s}$ ) was used to obtain sagittally oriented images. Thereby, the most prominent motion (head-feet and anterior-posterior) was kept in-plane. In-plane resolution was chosen to be slightly higher than the maximal PET resolution; higher spatial resolution was avoided for the sake of temporal resolution (at least 2 acquisitions per second to get images near the respiratory reference position).

To completely cover 1 respiratory cycle, 12 frames of each slice were acquired subsequently before moving on to the next slice; 36 slice positions in the left-to-right direction were acquired to cover the patient's thorax and upper abdomen, resulting in approximately $10-\mathrm{mm}$ resolution in this direction. This low resolution was accepted under the assumption that respiratory motion in the left-right direction has only a small amplitude. Before each frame, a navigator image of the diaphragm position was acquired in $0.06 \mathrm{~s}$. During the remainder of the PET acquisition time, only the navigator data were acquired (Fig. 2).
FIGURE 1. Overview of proposed method for motion correction. Dashed line shows standard PET reconstruction.

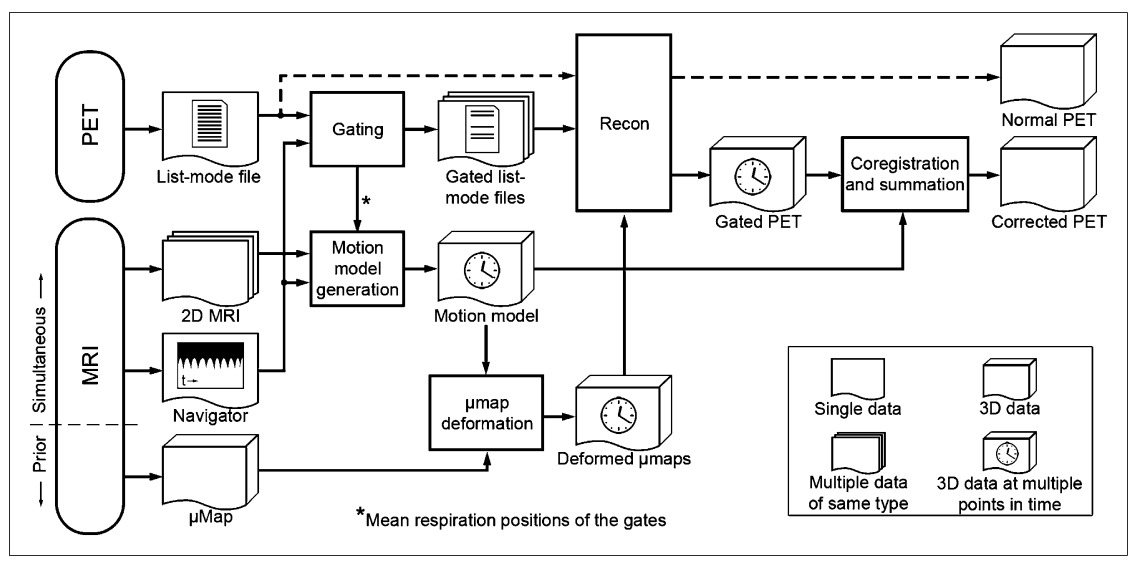




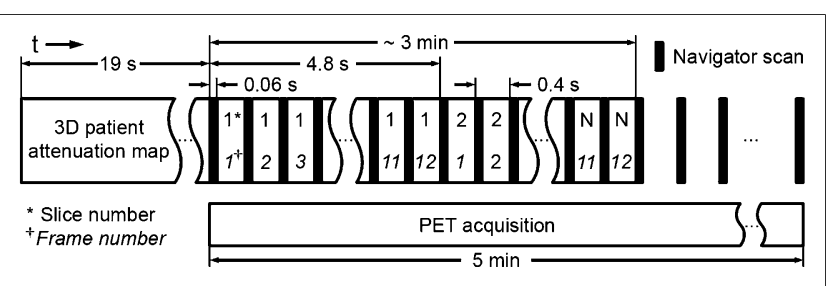

FIGURE 2. Timing of imaging procedure: patient $\mu$ Map (3D patient attenuation map) is acquired before simultaneous PET/MR examination for motion compensation. Twelve frames of each MR slice are measured before moving to next slice. Navigator scan is acquired between frames throughout PET scan as respiration signal.

From these data, the noncorrected, motion-corrected, and gated PET images (end-expiratory image of the 4 respiratory gates used for motion correction) were derived.

We decided to use a navigator instead of a respiration belt to minimize the effort needed for motion compensation. Although we could not find any direct comparison between external respiration tracking systems and MR navigators, the superiority of navigator-based triggering over external systems in the reduction of respiration-induced artifacts in MR imaging has been reported (25).

\section{Data Processing}

The following PET image datasets were prepared for analysis: the nongated uncorrected PET dataset, the dataset with motion turned off (phantom only), the gated dataset at the end-expiratory position, and the motion-corrected dataset. The first two did not need preprocessing and were directly reconstructed using the vendor-provided 3D ordered-subset expectation maximization algorithm (3 iterations, 21 subsets, 3-mm gaussian filter) with the appropriate $\mu$ Map. Creation of the motion-corrected PET image dataset (phantom and patients) required more extensive data processing. The retrospectively gated MR images in the end-expiratory position were obtained by executing only the first step of the motion correction procedure.

Data processing can be divided into 4 stages. The first is respiratory gating of list-mode data, including analysis of the navigator data to obtain a respiration signal; the second, a reordering of the 2D MR images and motion field generation; the third, the reconstruction of the gated PET images with spatially adapted $\mu$ Maps; and the fourth, a coregistration and summation of the gated PET images.

Respiratory Gating. Before the list-mode data could be sorted into respiratory gates, the data had to be synchronized with the MR-obtained respiration signal by parsing of the list-mode file for a synchronization tag that is inserted by the MR scanner at the beginning of the imaging sequence. The MR navigator data were acquired during the full PET acquisition and were used to define 4 respiratory gates with respect to the amplitude of diaphragm motion, dividing the range between its 0.05 and 0.95 quantiles into 4 equally sized intervals (to reduce the influence of outliers), and the complete PET list mode data were sorted into these 4 respiratory gates (4 gates were confirmed to be a good compromise between count statistics and positional accuracy in initial experiments [supplemental data]). The mean respiratory position within the respective gate interval was calculated for each gate and used as a reference position for the motion field generation.
Reordering of MR Data and Motion Field Generation. To obtain an MR basis for the motion fields, the 2D MR slices had to be reordered into 4D volumes (multiple 3D volumes of different time points in the respiratory cycle). Thus, for each slice and each gated list-mode frame, the MR image closest to the frame's mean respiratory position was inserted into the corresponding $3 \mathrm{D}$ volume, and the remaining 11 images were discarded (Fig. 3). The motion fields (a set of three 3D vector fields mapping the nonend-expiratory $3 \mathrm{D}$ volumes into the end-expiratory $3 \mathrm{D}$ volume) derived from the first $3 \mathrm{~min}$ of MR acquisition were calculated using a nonrigid demons registration algorithm (26) (mutual information criterion, parameters: $\alpha=2, \sigma_{\text {fluid }}=8, \sigma_{\text {diffusion }}=1$ ) and was used for the complete gated PET dataset.

PET Gated Image Reconstruction with Attenuation Correction. PET images were reconstructed using the same ordered-subset expectation maximization algorithm and same settings as before. In addition to the gated emission data, $\mu$ Maps matching the different respiratory positions were needed. On the Biograph mMR system, attenuation correction is split into a hardware part, compensating for the attenuation of the scanner hardware (hardcoded), and the patient attenuation. First, the MR image of the end-expiratory position was superposed on the Dixon dataset to confirm adequate alignment of the end-expiratory position in the MR data used for motion field generation and the $\mu$ Map. The motion fields were used to transform the end-expiratory patient $\mu$ Map to match the mean respiratory position of the gated listmode frames. This procedure corrects for motion between the different gates; however, a possible mismatch between endrespiratory PET and $\mu$ Map obtained from breath-hold MR remains uncorrected. The $\mu$ Map was also visually assessed to rule out any image artifacts.

Coregistration and Image Summation. Finally, the motioncorrected 3D PET image was obtained by coregistration of the gated PET image datasets using the motion fields, scaling each PET image according to the number of counts in the corresponding gated list-mode file and their summation on a pixel-by-pixel basis.

\section{Data Analysis}

A custom-written user interface in Matlab (The MathWorks) was used for the evaluation of line profiles drawn through the maxima of the lesions. From the obtained profiles, the following

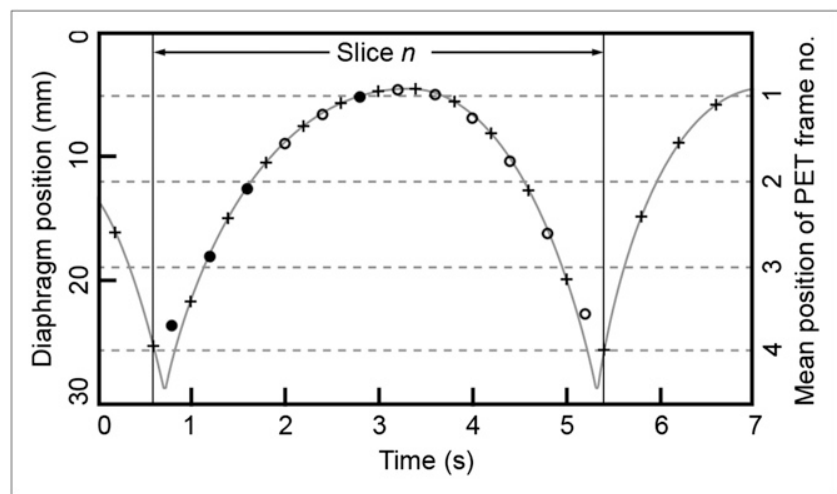

FIGURE 3. Head-feet position of diaphragm (gray line) is sampled by MR navigator every $0.4 \mathrm{~s}(+)$. Mean position of acquired image slices is estimated by linear interpolation of these values $(\bigcirc)$. Each slice is sampled 12 times, and slices closest to mean positions of PET frames are chosen for motion field creation $(\bullet)$. 
parameters were extracted: the mean slope steepness (calculated from the steepest parts of the rising and falling edges) and the full width at half maximum (FWHM). Profiles were drawn parallel to the direction of movement (phantom and patient study) and orthogonal to the direction of movement (phantom study only). The Syngo TrueD (Siemens Healthcare) software was used for the evaluation of 3D ellipsoid volumes of interest around the lesions. From this evaluation, the lesion's maximum intensity (maximum standardized uptake value $\left[\mathrm{SUV}_{\max }\right]$ for patient study), contrast (ratio between $\mathrm{SUV}_{\max }$ and mean intensity in a region of interest in the surrounding tissue), SNR (ratio between $S_{\text {U }}$ max and SD of the same region), and lesion volume were obtained. The software determines the lesion volume by regarding all voxels inside the ellipsoid having an SUV greater than $50 \%$ of the lesion's SUV $_{\max }$ as contributing to the lesion volume.

Mean values are provided together with the SD and were statistically compared using the Wilcoxon signed rank test (because of nonnormally distributed samples) in the patient study (SPSS Statistics, version 20; IBM Corp.). Because all 3 combinations of data were evaluated (uncorrected/gated, corrected/gated, corrected/ uncorrected), the effective significance level of $\alpha=0.05$ was adjusted according to a previously published method (27), yielding an adjusted significance level of $P=0.017$.

\section{RESULTS}

\section{Phantom Study}

The relative changes in lesion parameters in the uncorrected, gated, and corrected phantom images as compared with the images of the nonmoving phantom (reference standard) were assessed (Table 3). All values given are means over the phantom's 3 point sources. Both gating and motion correction are able to greatly reduce the negative impact of the motion, as compared with the uncorrected image. All results connected to image resolution such as FWHM and slope steepness orthogonal to the direction of movement were slightly worse for motion correction than gating, and the SNR was significantly better. Figure 4 shows the influence of the reconstruction on the image of 1 point source.

\section{Patient Study}

The changes in image parameters in 14 evaluated lesions in terms of mean, SD, and $P$ values are given in Table 4 for all 3 combinations of data. The complete data (values of

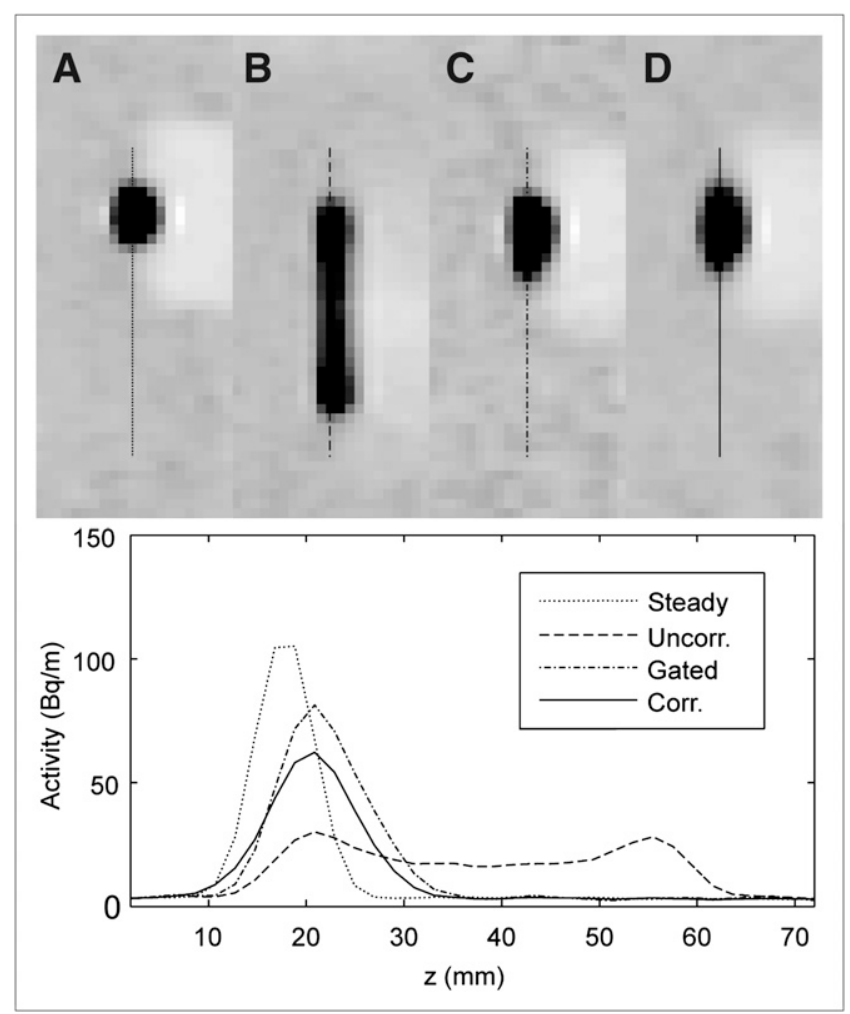

FIGURE 4. Axial profiles (head-feet direction) of phantom point source. (A) Point source of steady phantom. (B-D) Images of same source in moving phantom (uncorrected $[B]$, gated $[C]$, motion-corrected $[D]$ ) and corresponding line profiles. Contrast and delineation between cube and background activity is remarkably restored using motion correction information.

individual lesions) are available in Supplemental Tables 1 and 3 .

Comparison between corrected and uncorrected images showed a highly significant improvement in all image parameters $\left(P=0.001\right.$ for contrast, $\mathrm{SUV}_{\max }, \mathrm{FWHM}$, and slope steepness; $P=0.002$ for volume; and $P=0.003$ for SNR). Similar changes could be observed when uncorrected and gated images were compared, where changes in contrast, $\mathrm{SUV}_{\max }, \mathrm{FWHM}$, slope steepness, and lesion volume were large and highly significant $(P=0.001)$. In

TABLE 3

Percentage Change of Image Parameters in Phantom Study Compared with Steady Phantom (Reference Standard)

\begin{tabular}{|c|c|c|c|c|c|c|c|c|c|}
\hline \multirow[b]{2}{*}{ Image } & \multirow[b]{2}{*}{ Index } & \multirow[b]{2}{*}{ Contrast } & \multirow[b]{2}{*}{ Max } & \multirow[b]{2}{*}{ SNR } & \multirow[b]{2}{*}{ Volume } & \multicolumn{2}{|c|}{$\mathrm{HF}$} & \multicolumn{2}{|c|}{ AP } \\
\hline & & & & & & FWHM & Slope & FWHM & Slope \\
\hline \multirow[t]{2}{*}{ Uncorrected } & Mean & $-69.2 \%$ & $-78.6 \%$ & $-72.2 \%$ & $352,1 \%$ & $474.2 \%$ & $-77.9 \%$ & $0.3 \%$ & $-77.3 \%$ \\
\hline & SD & $6.2 \%$ & $18.6 \%$ & $0.2 \%$ & $28.9 \%$ & $33.7 \%$ & $3.9 \%$ & $8.4 \%$ & $2.3 \%$ \\
\hline \multirow[t]{2}{*}{ Gated } & Mean & $-23.2 \%$ & $-22.3 \%$ & $-54.4 \%$ & $22.0 \%$ & $33.1 \%$ & $-67.2 \%$ & $-0.2 \%$ & $-33.2 \%$ \\
\hline & SD & $1.3 \%$ & $0.5 \%$ & $2.5 \%$ & $11.1 \%$ & $4.4 \%$ & $3.4 \%$ & $4.3 \%$ & $8.5 \%$ \\
\hline \multirow[t]{2}{*}{ Corrected } & Mean & $-38.8 \%$ & $-38.5 \%$ & $-33.4 \%$ & $29.8 \%$ & $34.8 . \%$ & $-60.9 \%$ & $12.0 \%$ & $-55.8 \%$ \\
\hline & SD & $2.6 \%$ & $2.8 \%$ & $5.8 \%$ & $22.5 \%$ & $1.8 \%$ & $3.3 \%$ & $14.5 \%$ & $8.7 \%$ \\
\hline
\end{tabular}

$\mathrm{HF}=$ head-feet direction; $\mathrm{AP}=$ anterior-posterior direction; $\max =$ lesion maximum. 
TABLE 4

Mean Change of Lesion Parameters of 14 Evaluated Moving Lesions

\begin{tabular}{|c|c|c|c|c|c|c|c|}
\hline Comparison & Index & Contrast & SUV $_{\text {Max }}$ & SNR & FWHM & Slope & Volume \\
\hline \multirow[t]{3}{*}{ Uncorrected/gated } & Mean & $-28.1 \%$ & $-29.3 \%$ & $3.2 \%$ & $32.3 \%$ & $-50.3 \%$ & $80.0 \%$ \\
\hline & SD & $11.2 \%$ & $12.7 \%$ & $22.7 \%$ & $16.2 \%$ & $11.4 \%$ & $74.3 \%$ \\
\hline & $P$ & $0.001^{*}$ & $0.001^{*}$ & 0.944 & $0.001^{*}$ & $0.001^{*}$ & $0.001^{*}$ \\
\hline \multirow[t]{3}{*}{ Corrected/gated } & Mean & $-11.3 \%$ & $-10.7 \%$ & $27.0 \%$ & $3.9 \%$ & $-19.3 \%$ & $27.8 \%$ \\
\hline & $\mathrm{SD}$ & $11.2 \%$ & $11.3 \%$ & $20.3 \%$ & $7.1 \%$ & $12.0 \%$ & $63.3 \%$ \\
\hline & $P$ & $0.002^{\star}$ & $0.003^{*}$ & $0.004^{\star}$ & 0.059 & $0.001^{*}$ & 0.04 \\
\hline \multirow[t]{3}{*}{ Corrected/uncorrected } & Mean & $24.7 \%$ & $28.1 \%$ & $27.3 \%$ & $-20.6 \%$ & $66.7 \%$ & $-26.5 \%$ \\
\hline & $\mathrm{SD}$ & $14.7 \%$ & $13.6 \%$ & $28.5 \%$ & $8.9 \%$ & $31.1 \%$ & $19.3 \%$ \\
\hline & $P$ & $0.001^{*}$ & $0.001^{\star}$ & $0.003^{\star}$ & $0.001^{*}$ & $0.001^{*}$ & $0.002^{*}$ \\
\hline
\end{tabular}

*Statistically significant.

terms of SNR, the positive effects (increased $\mathrm{SUV}_{\max }$ ) and negative effects (increased noise) of the gating procedure cancel out each other and result in an insignificant change $(P=0.944)$. Comparing the end-expiratory-gate images with the proposed correction method shows that SNR is significantly increased, by $27.0 \%(P=0.004)$. However, similar to the phantom study, our method performed significantly worse in terms of contrast $(-11.3 \%, P=0.002)$, $\mathrm{SUV}_{\text {max }}(-10.7 \%, P=0.003)$, and slope steepness $(-19.3 \%$, $P=0.001$ ) (Fig. 5).

The effect of the different reconstruction techniques on static lesions was evaluated in 8 lesions (Table 2). Lesions in gated images showed a slight but significant increase in slope steepness compared with both uncorrected and corrected images, but at the cost of severely decreased SNR. No significant change between uncorrected and corrected images could be found in any parameter.

\section{DISCUSSION}

The proposed MR-based respiratory motion correction method for PET data worked well in both phantom and patient measurements. In reducing motion blur and restoring lesion intensity, the proposed method showed potential similar to (though slightly less than) end-expiratory gating, but without loss in count statistics. It was demonstrated that MR-based correction of respiratory motion in thoracic and abdominal tumors is clinically feasible and does not cause an excessive increase in examination time. Furthermore, it does not require any attachment of gating devices to the patient, thus increasing patient comfort and reducing technician radiation exposure. The visibility of moving lesions was clearly enhanced and uptake quantification was significantly improved, whereas negative effects on nonmoving lesions were insignificant. One major image improvement was the $28.1 \%$ gain in lesion $\mathrm{SUV}_{\text {max }}$, which has 2 consequences. First, lesion contrast was increased by $24.7 \%$, which is beneficial in the detection of small lesions with diameters of less than $10 \mathrm{~mm}$ (lesion A8; Fig. 6) or lesions near large structures with heavy uptake (lesion C2, Supplemental Fig. 2). Sec- ond, the increased lesion intensity provides more accurate information about the true radiotracer uptake of the lesion. An average reduction in FWHM of $20.6 \%$ along the headfeet direction was shown. The FWHM for lesions A5, A8, and B1 could be decreased to less than $6 \mathrm{~mm}$, which is already close to the physical resolution of the scanner (28). Slope steepness was significantly increased, by $66.7 \%$ on average.

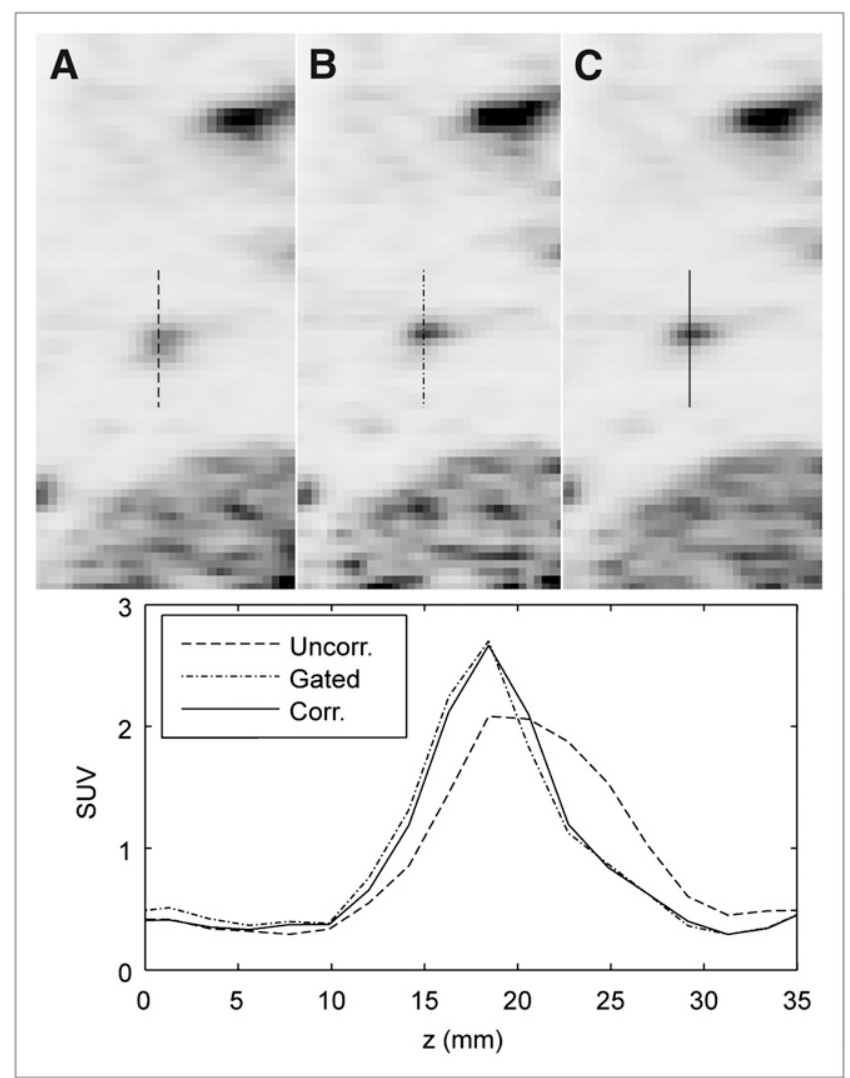

FIGURE 5. Profiles in head-feet direction through lesion $A 1$ for uncorrected PET image (A), gated image (B), and motion-corrected image (C). Sharper delineation and increase in lesion intensity are clearly visible. Lesion profile from gated image shows highest slope steepness, but at cost of increased background noise. 


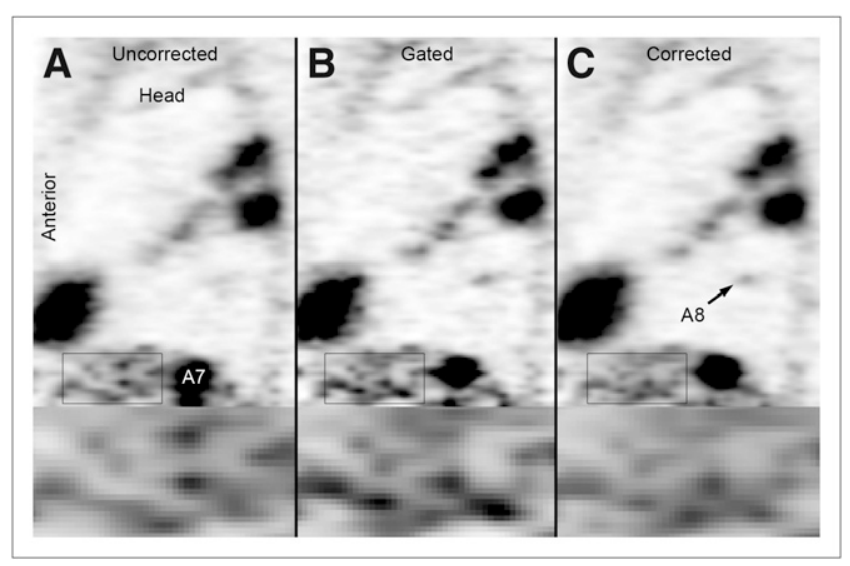

FIGURE 6. Comparison of uncorrected (A), gated (B), and corrected $(C)$ sagittal PET image slice featuring lesions $A 7$ and $A 8$ in patient $A$. Multiple lesions and inflammatory areas with increased ${ }^{18} \mathrm{~F}-\mathrm{FDG}$ uptake in lung show enhanced delineation. Lesion A7 in spleen is much smaller after correction. Lung metastasis A8 (confirmed by CT scan) is clearly enhanced in gated and corrected image. Increased background noise is clearly visible in gated image (zoomed region at bottom).

The methodology introduced and evaluated in this work provides patient data on simultaneous thoracic and abdominal imaging, thereby complementing prior work in brain imaging (18), simulated and phantom data in thoracic and abdominal imaging $(19,21,22)$, and animal studies (23). Details and parameters of the proposed motion correction method were chosen to be feasible in routine clinical imaging. One main goal was to keep the MR time needed exclusively for motion correction as low as possible. MR images for generation of the motion map were acquired only during the first $3 \mathrm{~min}$ or so of the PET scan. During the remainder of the PET acquisition, only navigator data were acquired for detection of the respiratory position. This navigator is compatible with many diagnostic MR sequences that can run simultaneously. The 2D multislice-imaging approach over several breathing periods together with the 1-dimensional navigator motion detection during the whole PET scan assumes that the tissue deformation is reproducible from respiration period to respiration period, that is, once the motion field is available for a specific navigator position, the same model can be used for the same navigator position at any point in time. Thus, inter- and intracycle variations, such as through gross movement of the body, cannot be captured in full detail, as discussed by King et al. (20).

Real-time 4D MR imaging with sufficient spatial and temporal resolution as suggested by King et al. (20) is not feasible, since receive coils with the required amount of channels (and thus such a high acceleration factor) are not available on the Biograph mMR. Methods for the acquisition of 4D MR images acquired over multiple respiratory cycles were previously described $(19,29)$. A previously described 3D imaging approach (29) required heavy oversampling of the k-space to obtain several respiration frames without perceivable motion artifacts, thus requiring long scan times of more than 10 min per bed position, likely too long for routine imaging, in which motion correction of 2 or 3 bed positions is necessary. Dikaios et al. compared the creation of 4D MR images on the basis of state-of-the-art $3 \mathrm{D}$ and $2 \mathrm{D}$ techniques and suggested using a 2D multislice technique in terms of image contrast, spatial resolution, and temporal resolution (19). The acquisition time per image frame could be further decreased using parallel imaging. The accuracy of the motion estimation might also be increased at areas of sharp transitions in the motion fields (e.g., between liver and spine) by tagging sequences $(22,23)$. Motion is visualized intrinsically by the tagging lines, but at the cost of losing all diagnostically useful information from the MR imaging data used for motion estimation as well as spatial resolution (limited by tag line distance). The relative merit of the 2 approaches remains to be seen, optimally with a head-to-head comparison in the same patients. However, the diagnostically useful T1-weighted images can potentially be applied for the generation of patient $\mu$ Maps at different respiration positions.

Although improving significantly on uncorrected nongated image acquisition, there was still an improvement in contrast and uptake quantification when going from motioncorrected to gated images (albeit with unwanted degradation of count statistics). Some of the influencing factors include number of respiratory gates and postreconstruction summation of images. During the end-expiratory phase of the respiratory cycle, used as the reference gate within this work, there is less movement than during the other phases. Because other gates have worse image characteristics than this "ideal" gate, a part of the decreased contrast in the motion-corrected images is likely to stem from in-gate blurring rather than from the MRbased gate combination procedure.

As previously proposed (9), we applied amplitude-based gating in our method since it is considered to better compensate for motion. The suggested number of 6-8 respiratory gates was reduced to 4 because of the reduced tracer activity in our case (supplemental data). In future routine imaging, less uptake time or a longer scan duration per bed position may allow an increase in respiratory gates. In this study, the timing of PET/MR imaging was restrained by the ethics approval allowing for PET/MR imaging only after clinical PET/CT.

Instead of using postreconstruction registration through the help of the MR-derived motion fields, application of the motion fields to the list-mode data before or during reconstruction is feasible. This approach has been used in CT-based motion correction (15), simulated PET/MR scenarios $(19,20)$, phantom studies $(21,22)$, and animal studies (23). Since signal-to-noise considerations do not play a role anymore, more finely grained motion fields would be feasible: instead of distributing all counts into 4 discrete respiratory gates, a continuous respiratory position scale could be used-for example, derived from the discrete MR datasets by interpolation. This method could also re- 
duce the blurring influences of the multiple reconstruction processes involved in the motion correction procedure and could explain the better performance of the gating procedure in terms of contrast, intensity maximum, and slope steepness. Implementation of a motion-sensitive reconstruction algorithm for the Biograph mMR system was outside the scope of this work but would be completely compatible with the proposed MR method for motion field generation.

A limitation of the presented method is the use of a $2 \mathrm{D}$ MR sequence for motion correction that might not be of diagnostic interest, blocking $3 \mathrm{~min}$ of the simultaneous PET/MR examination time. Thus, an acceleration of the MR sequence or application of sequences useful for diagnostics and motion correction at the same time would be beneficial.

The motion correction approach may be extended from tumor imaging to cardiac or vessel imaging. To cope with the then more relevant flow-related changes in the MR images, adapted registration algorithms may be needed.

\section{CONCLUSION}

Simultaneous PET/MR imaging offers the possibility of significantly enhanced PET image quality and improved radiotracer uptake quantification in thoracic and abdominal tumor PET. The proposed method was shown to work well in a time-efficient and convenient manner (no attachment of additional equipment to the patient), allowing for easy integration into clinical routine imaging. Motion correction will be most beneficial in the detection of small lesions with low contrast from surrounding tissue and in treatment response evaluations based on uptake quantification.

\section{DISCLOSURE}

The costs of publication of this article were defrayed in part by the payment of page charges. Therefore, and solely to indicate this fact, this article is hereby marked "advertisement" in accordance with 18 USC section 1734. Financial research support was provided by the Medical Faculty of the University of Tübingen (fortüne program, grant 1891-0-0), Deutsche Forschungsgemeinschaft SFB 656 Münster (project C6), and the Herzzentrum Münster e.V. (travel grant). No other potential conflict of interest relevant to this article was reported.

\section{ACKNOWLEDGMENTS}

We thank Carsten Groeper and Gerd Zeger for image acquisition and Brigitte Gückel for administration of the scientific study.

\section{REFERENCES}

1. Judenhofer MS, Wehrl HF, Newport DF, et al. Simultaneous PET-MRI: a new approach for functional and morphological imaging. Nat Med. 2008;14:459-465.

2. Osman MM, Cohade C, Nakamoto Y, Marshall LT, Leal JP, Wahl RL. Clinically significant inaccurate localization of lesions with PET/CT: frequency in 300 patients. J Nucl Med. 2003;44:240-243.
3. Seppenwoolde Y, Shirato H, Kitamura K, et al. Precise and real-time measurement of 3D tumor motion in lung due to breathing and heartbeat measured during radiotherapy. Int J Radiat Oncol Biol Phys. 2002;53:822-834.

4. Nakamoto Y, Chin BB, Cohade C, Osman M, Tatsumi M, Wahl RL. PET/CT artifacts caused by bowel motion. Nucl Med Commun. 2004;25:221-225.

5. Erdi YE, Nehmeh SA, Pan T, et al. The CT motion quantitation of lung lesions and its impact on PET-measured SUVs. J Nucl Med. 2004;45:12871292.

6. Papathanassiou D, Becker S, Amir R, Menéroux B, Liehn JC. Respiratory motion artefact in the liver dome on FDG PET/CT: comparison of attenuation correction with CT and a caesium external source. Eur J Nucl Med Mol Imaging. 2005;32:1422-1428.

7. Schwarz AJ, Leach MO. Implications of respiratory motion for the quantification of 2D MR spectroscopic imaging data in the abdomen. Phys Med Biol. 2000; 45:2105-2116.

8. McLeish K, Hill DL, Atkinson D, Blackall JM, Razavi R. A study of the motion and deformation of the heart due to respiration. IEEE Trans Med Imaging. 2002;21:1142-1150.

9. Dawood M, Büther F, Stegger L, et al. Optimal number of respiratory gates in positron emission tomography: a cardiac patient study. Med Phys. 2009;36: 1775-1784.

10. Suramo I, Päivänsalo M, Myllylä V. Cranio-caudal movements of the liver, pancreas and kidneys in respiration. Acta Radiol Diagn (Stockh). 1984;25:129-131.

11. Nehmeh SA, Erdi YE, Ling CC, et al. Effect of respiratory gating on quantifying PET images of lung cancer. J Nucl Med. 2002;43:876-881.

12. Dawood M, Lang N, Jiang X, Schäfers KP. Lung motion correction on respiratory gated 3-D PET/CT images. IEEE Trans Med Imaging. 2006;25:476-485.

13. Dawood M, Buther F, Jiang X, Schafers KP. Respiratory motion correction in 3-D PET data with advanced optical flow algorithms. IEEE Trans Med Imaging. 2008;27:1164-1175.

14. Bai W, Brady M. Motion correction and attenuation correction for respiratory gated PET images. IEEE Trans Med Imaging. 2011;30:351-365.

15. Lamare F, Ledesma Carbayo MJ, Cresson T, et al. List-mode-based reconstruction for respiratory motion correction in PET using non-rigid body transformations. Phys Med Biol. 2007;52:5187-5204.

16. Dikaios N, Fryer TD. Improved motion-compensated image reconstruction for PET using sensitivity correction per respiratory gate and an approximate tube-ofresponse backprojector. Med Phys. 2011;38:4958-4970.

17. Pichler BJ, Wehrl HF, Judenhofer MS. Latest advances in molecular imaging instrumentation. J Nucl Med. 2008;49(suppl):5S-23S.

18. Catana C, Brenner T, van der Kouwe A, et al. MRI-assisted PET motion correction for neurologic studies in an integrated MR-PET scanner. J Nucl Med. 2011;52:154-161.

19. Dikaios N, Izquierdo-Garcia D, Graves MJ, Mani V, Fayad ZA, Fryer TD. MRIbased motion correction of thoracic PET: initial comparison of acquisition protocols and correction strategies suitable for simultaneous PET/MRI systems. Eur Radiol. 2012;22:439-446.

20. King AP, Buerger C, Tsoumpas C, Mardsen PK, Schaeffter T. Thoracic respiration motion estimation from MRI using a statistical model and a 2-D image navigator. Med Image Anal. 2012;16:252-264.

21. Tsoumpas C, Mackewn JE, Halsted P, et al. Simultaneous PET-MR acquisition and MR-derived motion fields for correction of non-rigid motion in PET. Ann Nucl Med. 2010;24:745-750.

22. Guérin B, Cho S, Chun SY, et al. Nonrigid PET motion compensation in the lower abdomen using simultaneous tagged-MRI and PET imaging. Med Phys. 2011;38:3025-3038.

23. Chun SY, Reese TG, Ouyang J, et al. MRI-based nonrigid motion correction in simultaneous PET/MRI. J Nucl Med. 2012;53:1284-1291.

24. Martinez-Möller A, Souvatzolgou M, Delso G, et al. Tissue classification as a potential approach for attenuation correction in whole-body PET/MRI: evaluation with PET/CT data. J Nucl Med. 2009;50:520-526.

25. Kim BS, Kim JH, Choi GM, et al. Comparison of three free-breathing T2weighted MRI sequences in the evaluation of focal liver lesions. AJR. 2008;190:W19-W27.

26. Thirion JP. Image matching as a diffusion process: an analogy with Maxwell's demons. Med Image Anal. 1998;2:243-260.

27. Cupples LA, Heeren T, Schatzkin A, Colton T. Multiple testing of hypotheses in comparing two groups. Ann Intern Med. 1984;100:122-129.

28. Delso G, Fürst S, Jakoby B, et al. Performance measurements of the Siemens mMR integrated whole-body PET/MR scanner. J Nucl Med. 2011;52:1914-1922.

29. Ablitt NA, Gao J, Keegan J, Stegger L, Firmin DN, Yang GZ. Predictive cardiac motion modeling and correction with partial least squares regression. IEEE Trans Med Imaging. 2004;23:1315-1324. 